\title{
MRF-based image segmentation using Ant Colony System
}

\author{
Salima Ouadfel and Mohamed Batouche \\ Computer Vision Group, LIRE Laboratory \\ Computer Science Department, Mentouri University \\ Route de Ain El-Bey, Constantine, 25000, Algeria \\ E-mail: \{souadfel, batouche\} wissal.dz
}

Received 28 February 2003; accepted 9 July 2003

\begin{abstract}
In this paper, we propose a novel method for image segmentation that we call ACS-MRF method. ACS-MRF is a hybrid ant colony system coupled with a local search. We show how a colony of cooperating ants are able to estimate the labels field and minimize the MAP estimate. Cooperation between ants is performed by exchanging information through pheromone updating. The obtained results show the efficiency of the new algorithm, which is able to compete with other stochastic optimization methods like Simulated annealing and Genetic algorithm in terms of solution quality.
\end{abstract}

Key Words: Image Analysis, Image Segmentation, Markov Random Field, Ant Colony Optimization, Ant Colony System, Local search.

\section{Introduction}

Image segmentation is a low level image processing task that aims at partitioning an image into regions in order that each region groups contiguous pixels sharing similar attributes (intensity, color, etc.). It is a very important process because it is the first step of the image understanding process, and all others steps, such as feature extraction, classification and recognition, depend heavily on its results. Image segmentation has been the subject of intensive research, and a wide variety of image segmentation techniques have been reported in the literature. A good review of these methods can be found in [22]. Among them, Markov random field (MRF) is one of the most frequently utilized [2, 4, 7, 15, 23, 25, 26, 29, $32,33,37]$. The MRF-based image segmentation method is a process seeking the optimal labeling of the image pixels. A labeling process consist of accurately labeling the image pixels with a group of given labels. A label set represents the pattern classes in the image. Through a process based on local interactions among pixels, MRF allows the label selection of a pixel to be conditioned explicitly on the local interaction between the pixel and its neighbors within a well-defined neighborhood system without involving all the pixels of the image.

The image is segmented by maximizing the a posteriori probability (MAP) of the labeling space given the image data [31]. The MRF-MAP framework involves solving an energy maximization (or minimization) problem. However this maximization is combinatorial and the energy function is usually non convex and may exhibits many local minima in the solution space. The often used methods for solving such

Correspondence to: souadfel@wissal.dz, batouche@wissal.dz

Recommended for acceptance by Enric Martí

ELCVIA ISSN: $1577-5097$

Published by Computer Vision Center / Universitat Autonoma de Barcelona, Barcelona, Spain 
combinatorial problem are the iterated conditional method (ICM) [12], the simulated annealing (SA) [28] and the genetic algorithm (GA) [9, 23].

ICM is a deterministic optimization method proposed by Besag [12] and based on a descent gradient strategy. For each pixel, the label which gives the large decrease of the energy function is chosen. ICM converge quickly to a local solution and the solution quality depends much on the initial partition [31, 35]. SA is a stochastic algorithm based on the classical Metropolis method and introduced in computer vision by Geman and Geman [28]. The SA algorithm converges to global minima of the energy function but with an exponential time [30]. GA developed by Holland [14], is a stochastic optimization method that mimic the principles of natural evolution. Unlike the above-mentioned methods, which operate on a single configuration, GA is a population-based method, which operates on a population of individuals called chromosomes. Each chromosome represents a solution to the given problem. The chromosomes evolve through successive generations and two genetic operators are used to produce offspring: crossover and mutation [6]. The resulting offspring update the population according to a fittest function. Applications of the GA in the image segmentation can be found in $[9,10,13,36]$.

Ant Colony Optimization (ACO) metaheuristic [17, 21] is a recent population-based approach inspired by the observation of real ants colony and based upon their collective foraging behavior. In ACO, solutions of the problem are constructed within a stochastic iterative process, by adding solution components to partial solutions. Each individual ant constructs a part of the solution using an artificial pheromone, which reflects its experience accumulated while solving the problem, and heuristic information dependent on the problem. In this paper, we applied the concept of ACO for discrete optimization in MRF-based image segmentation. Our main motivation is that ACO metaheuristic has been successfully applied to several NP-hard combinatorial optimization problems and has been shown to be competitive against conventional optimization methods like GA and SA [18]. We have designed a constructive algorithm called ACS-MRF algorithm, in which a colony of artificial ant searches for an optimal labeling of image pixels that maximizes the MAP estimate. Individual ant constructs a candidate partition, by a relaxation labeling with respect to the contextual constraints. After some iterations, the partition representing the optimum value of the energy function is obtained. Experimental results are provided to compare the ACS-MRF method with the SA and GA. The results show that the ACS-MRF based image segmentation yields good quality solution comparable to SA and GA.

Consequently and in order to be self-contained, the rest of the paper is organized as follows. Section 2 presents a brief review on image modeling using MRF. Section 3 describes ACO algorithm and the ACS algorithm. Section 4 investigates the application of ACS for MRF-based image segmentation. In section 5 we present the experimental results and finally a conclusion is drawn in section 6 .

\section{Image Modeling using Markov Random Field}

The MRF was introduced in image analysis by Geman and Geman [28]. MRF theory provides a tool for modeling a vision problem within the Baye's framework using spatial continuity. MRF is a stochastic process in which spatial relations within the image are included in the labeling process through statistical dependence among neighboring pixels. This section briefly describes the MRF model for image segmentation. The image pixels are indexed by a rectangular lattice $S$ and each pixel $s$ is characterized by the gray level $y_{s}$ from the set $y=\left\{y_{s}: s \in S\right\}$. The labeling process consists of accurately labeling each pixel $s \in S$ with a class label representing the pattern class in the image. A label set is defined as $\Lambda=\{1, \ldots C\}$, where $C$ is the total number of the pattern classes in the image. A labeling is denoted by $x=\left\{x_{s}: x_{s} \in \Lambda, s \in S\right\}$ where $x_{s}=l$ indicates that that the class label $l$ is assigned to the pixel s.

The goal is to find the labeling $\hat{x}$ of the image, which is the estimation of the true but unknown labeling $\mathbf{x}$. According to the MAP estimate [28] we have 


$$
\widehat{x}=\underset{x}{\arg \max }\{P(x \mid y)\}
$$

According to Baye's theorem [27], we have

$$
P(x \mid y)=\frac{1}{P(y)} P(y \mid x) \cdot P(x)
$$

where $P(x)$ is the prior density of the labeling $x$ and $P(y \mid \mathrm{x})$ is the conditional probability density of the image $y$.

The prior probability of the image $P(y)$ is independent of the labeling $x$, we have

$$
\widehat{x}=\arg \max _{x}\{P(y \mid x) \cdot P(x)\}
$$

We assumed that the image data are obtained by adding an identical independently distributed (i.i.d.) Gaussian noise. We have

$$
P\left(y_{s} \mid x_{s}\right)=\frac{1}{\sqrt{2 \pi \sigma_{x s}^{2}}} \operatorname{Exp}\left\{-\frac{\left(y_{s}-\mu_{x_{s}}\right)^{2}}{2 \sigma_{x_{s}}^{2}}\right\}
$$

Based on the conditional independence assumption of $y$, the conditional density $P(y \mid x)$ takes the form of

$$
P(y \mid x)=\prod_{s \in S} P\left(y_{s} \mid x_{s}\right)
$$

Therefore, $P(y \mid x)$ can be written as follows.

$$
P(y \mid x)=\prod_{s \in S}\left(\frac{1}{\sqrt{2 \pi}} \operatorname{Exp}\left\{-\frac{\left(y_{s}-\mu_{x s}\right)^{2}}{2 \sigma_{x s}^{2}}-\log \left(\sigma_{x s}\right)\right\}\right)
$$

which can be written as

$$
P(y \mid x)=\frac{1}{(2 \pi)^{\frac{S S}{2}}} \operatorname{Exp}\left[-\sum_{s \in S}\left\{\frac{\left(y_{s}-\mu_{x_{s}}\right)^{2}}{2 \sigma_{x s}^{2}}+\log \left(\sigma_{x_{s}}\right)\right\}\right]
$$

The prior model is based on 2-D MRF and assumes that the adjacent pixels are likely to have the same class label. We consider the neighboring system $N=\left\{N_{s}, s \in S\right\}$, where $N_{s}$ is the set of pixels neighboring s. $x$ is a particular realization of a random field X, which is an MRF on $S$ with respect to a neighboring system $N$, if for all $x \in \Omega$, 


$$
P(x)>0 \text { and } P\left(x_{s} \mid x_{s^{\prime}}, s \neq s^{\prime}, s^{\prime} \in S\right)=P\left(x_{s} \mid x_{s^{\prime}}, s^{\prime} \in N_{s} \backslash \mathbf{s}\right)
$$

where $\Omega$ is the set of all possible labeling and $N_{s} \backslash \mathrm{s}$ indicates the neighborhood $N_{s}$ excluding the pixel s.

According to the Hammersley-Clifford theorem [12], the prior model $P(x)$ is given by a Gibbs distribution with respect to the neighboring system $N . P(x)$ takes the form of

$$
P(x)=\frac{1}{Z} \exp \left\{-\sum_{c \in C} V_{c}(x)\right\}
$$

where $\mathrm{Z}$ is the normalization function, $V_{c}(x)$ is the potential function for clique $\mathrm{c}$ and $\mathrm{C}$ is the set of all cliques over the image. A clique is a set of pixels that are neighbors of one another. In this paper we consider only the pair-site clique potentials of 8-neighborhood system, with the form $V\left(x_{1}, x_{2}\right)=-\beta$ if $x_{1}=x_{2}$ and 0 otherwise. $\beta$ is a positive parameter and the larger $\beta$, the larger is the influence of the neighboring pixels.

From (7) and (9), we can write the posterior probability

$$
P(x \mid y) \propto \exp \{-U(x)\}
$$

where the energy function $U(x)$ has the form

$$
U(x)=\left\{\sum_{s \in S} \frac{\left(y_{s}-\mu_{x s}\right)^{2}}{2 \sigma_{x s}^{2}}+\sum_{s \in S} \log \left(\sigma_{x s}\right)+\sum_{c \in C} V_{c}(x)\right\}
$$

The problem of finding the MAP estimate of the segmentation is expressed as the minimization of the posterior energy function. This task is usually a hard optimization problem because the number of possible label configurations is generally very large and moreover, the energy function may contains local minima. This paper describes a new effective approach for the minimization of the energy function, following the concept of Ant Colony Optimization and strongly based on Ant Colony System.

\section{Ant Colony System}

Ant Colony Optimization (ACO) is a population-based approach first designed by Marco Dorigo and coworkers [17] and inspired by the foraging behavior of ant colonies. Individuals ants are simple insects with limited memory and capable of performing simple actions. However, the collective behavior of ants provides intelligent solutions to problems such as finding the shortest paths from the nest to a food source. Ants foraging for food lay down quantities of a volatile chemical substance named pheromone, marking their path that it follows. Ants smell pheromone and decide to follow the path with a high probability and thereby reinforce it with a further quantity of pheromone. The probability that an ant chooses a path increases with the number of ants choosing the path at previous times and with the strength of the pheromone concentration laid on it $[18,24]$.

This concept has been applied to hard combinatorial optimization problems by creating a population of artificial ants that searches for optimal solutions according to the problem's constraints. In the ACO metaheuristic, artificial ants are defined as simple agents that repeatedly construct candidate solutions by 
adding components to partial solutions. Partial solutions are seen as the states of the construction process and the ant moves from one state to another until a complete solution is built. Each ant, moving from state $i$ to the state $j$ is probabilistically guided by two measures: an artificial pheromone trail $\tau(\mathrm{i}, \mathrm{j})$ representing experience gathered by ants in previous iteration when choosing this move and a heuristic information $\eta(i, j)$ that represents a priori information of the given problem. Each ant updates pheromone trails after having construct a complete solution, and the amount of pheromone deposited is a function of the quality of the solution constructed. The goal of this update process is the increasing the probability of choosing the moves that were part of good solutions, while decreasing all others. ACO algorithms have been successfully applied to diverse combinatorial optimization problems including symmetric and asymmetric traveling salesman [18, 19], quadratic assignment [8,34], graph-coloring [5], vehicle routing [3], telecommunication networks [11] and sequential ordering [16]. A good review of these algorithms can be found in [21].

ACS is one of the best implementation of ACO for the TSP problem. ACS introduced a particular use of pheromone trails. Pheromone trails are used for exploration and exploitation. Exploration allowed the construction of new solutions by choosing in a probabilistic way the components of a solution: a higher probability is given to components with a strong pheromone trail. Exploitation is based on the choice of the components with a strong pheromone trail and a decreasing cost. Also the pheromone is updated in two stages. A local pheromone updating rule is applied after an ant has built a candidate solution to the problem. Once all ants have built solutions, only the so-far best solution is used to globally increase the pheromone trail. The local updating has a diversification effect: every time an ant chooses a component of the solution the quantity of pheromone trail associated to this choice is decreased and it becomes less attractive that others. On the other hand, the global updating has an intensification effect because it permits the intensification of the search in the neighborhood of the best solution computed during the next iteration. The reader is referred to [20] for amore detailed description of the ACS algorithm.

\section{ACS Algorithm for MRF Image Segmentation}

In this paper, we applied the concept of ACS algorithm for discrete optimization in MRF-based image segmentation. We have designed a constructive algorithm called ACS-MRF in which a colony of artificial ants searches for a globally optimum solution defined as a correct labeling of image pixels with respect to the contextual constrains. An individual ant in turn traces a solution in a solution space made up of all possible labeling of image pixels. Each component of a solution is a pair $(s, l)$ representing the assignment of the class label $l$ to a pixel $s$. In ACS-MRF, $K$ ants explore the image in parallel, and this process is repeated for $M$ iterations. During each iteration, ants construct complete solutions communicating only indirectly via pheromone updates. The artificial pheromone trail is numeric information encoded as a matrix of dimension $(N, C)$ where $N$ is the number of image pixels and $C$ is the total number of the pattern classes in the image. The decisional basis for the solution construction step of an ant is provided by the artificial pheromone trail $\tau(s, l)$ associated to the pair $(s, l)$, which represents the desirability of setting $x_{s}=l$ in a solution, and made available by previous attempts of other ants. Once each ant has built a complete solution, this is improved using a local search method in order to enhance the quality of the solutions found during the search process. Finally, the pheromone trails are updated locally during solutions construction and globally at the end of each iteration according to the quality of the best solution found from the beginning of the labeling process. The algorithm stop iterating when a maximum of number of iterations has been performed.

Figure 1 shows a pseudo-code for the complete ACS-MRF algorithm. More details of the algorithm are described in the following subsections.

In figure $1, K$ is the number of ants, and $x^{k}$ is a solution constructed by ant $k$, where $k=1 \ldots K . x^{i b}$ denotes the iteration best solution, and $x^{g b}$ denotes the best solution found since the start of the algorithm. 


\subsection{Pheromone trail initialization}

In ACS-MRF we initialize all pheromone values $\tau$ for pairs $(s, l)$ with a value $\tau_{0}=1 /\left(N . U\left(x^{0}\right)\right)$, where $x^{0}$ is an initial solution created by a greedy heuristic. Image pixels are treated in order, and for each one is assigned the locally best possible class label that minimizes the local energy function given by

$$
U_{s}\left(x_{s}=l \mid y_{s}, x_{s^{\prime},} s^{\prime} \in N_{s} \backslash s\right)=\frac{\left(y_{s}-\mu_{l}\right)^{2}}{2 \sigma_{1}^{2}}+\log \left(\sigma_{l}\right)+\sum_{c: s \in c} V_{c}(x)
$$

The first two terms of $U_{s}$ represent the binding between the gray level at pixel $s$ and the segmentation; the last term indicated the smoothness constraints in the prior model.

\subsection{Solution construction}

ACS-MRF goal is to find the optimal labeling that minimizes the energy function given in (11). $K$ ants build possible labeling in parallel. The construction of a solution involves two steps. First, a pixel has to be chosen and next a class label has to be assigned to it. Each ant is initially put on a random chosen pixel and then iteratively moved from pixel to pixel until all image pixels have been treated. For the second step the pheromone trails are used, $\tau(s, l)$ referring to the desire of assigning class label $l$ to pixel $s$. The decision over which class label $l$ is assigned to pixel $s$ is made by the following pseudo-random-proportional rule according to [20]. Let $q$ a random number uniformly distributed in $[0,1]$ and $q_{0}$ a fixed probability.

- If $\mathrm{q} \leq q_{0}$ then, the ant chooses to the pixel $s$ the class label $l$ for which the pheromone trail $\tau(s, l)$ is highest.

$$
l=\underset{u \in \Lambda}{\arg \max } \tau(s, u)
$$

- Otherwise a random decision is made in which a class label $l=1, \ldots . C$ is chosen with probability $P(s, l)$.

$$
P(s, l)=\frac{\tau(s, l)}{\sum_{u \in \Lambda} \tau(s, u)}
$$

The parameter $q_{0}$ controls the relative importance of exploitation of already accumulated knowledge reflected in the pheromone trails versus the exploration of the new solutions.

\subsection{Local pheromone update}

While building a solution, the ant chooses a class label $l$ to a pixel $s$ and changes the amount of pheromone trail of the pair $(s, l)$ by applying the following local pheromone update:

$$
\tau^{\text {new }}(s, l)=(1-\rho) . \tau^{\text {old }}(s, l)+\rho . \tau_{0}
$$


Algorithm 1 ACS for MAP-MRF image segmentation:

Create initial solution $x^{0}$ by a greedy heuristic.

Set $\tau_{0}=1 /\left(N . U\left(x^{0}\right)\right)$.

FOR each pair $(s, l)$ Set $\tau(s, l)=\tau_{0} \quad$ END-FOR

\section{REPEAT}

FOR each ant $k=1, \ldots \ldots, K$ DO

FOR each pixel $s \in S$ DO

WITH probability $q_{0}$ :

$$
l=\underset{u \in \Lambda}{\arg \max } \tau(s, u)
$$

ELSE

Choose $l$ randomly from the labels set $\Lambda$ with probability $\frac{\tau(s, l)}{\sum_{u \in \Lambda} \tau(s, u)}$

$$
x_{s}^{k}=l
$$

\section{END-FOR}

\section{END-FOR}

FOR each ant $k=1, \ldots ., K$ DO

FOR each pixel $s \in S$ DO

$$
\tau(s, l)=(1-\rho) . \tau(s, l)+\rho \cdot \tau_{0}, \text { where } x_{s}^{k}=l
$$

\section{END-FOR}

\section{END-FOR}

FOR each ant $k=1, \ldots ., K$ DO

Locally improve $x^{k}$

Evaluate $x^{k}$ by computing $U\left(x^{k}\right)$

\section{END-FOR}

$$
\begin{aligned}
& x^{i b}=\underset{x=x^{1}, \ldots, x^{K}}{\operatorname{argmin}}(U(x)) \\
& \text { IF } U\left(x^{i b}\right)<U\left(x^{g b}\right) \text { THEN } x^{g b}=x^{i b}
\end{aligned}
$$

FOR each pixel $s \in S$ DO

FOR each class label $l \in \Lambda$ DO

$$
\begin{gathered}
\tau(s, l)=(1-\alpha) \cdot \tau(s, l)+\alpha \cdot \Delta \tau(s, l) \\
\Delta \tau(s, l)= \begin{cases}\frac{1}{U\left(x^{g b}\right)} & \text { if }(s, l) \in x^{g b} \\
0 & \text { otherwise }\end{cases}
\end{gathered}
$$

\section{END-FOR}

\section{END-FOR}

UNTIL $M$ iterations are performed. 
where $\rho$ is a parameter in ]0,1[, which represents the evaporation of the pheromone trail as in the behavior of the real ants and $\tau_{0}$ is the initial pheromone value.

The effect of local updating is to decrease the amount of the pheromone value associated to the pair $(s, l)$ used by an ant in order to reduce it's attractivity for the other ants building solutions in parallel.

\subsection{Global pheromone update}

When all ants have built a solution, the best solution corresponding to the $\min \left(U\left(x^{k}\right)\right)$ is recorded. The pheromone trails are then modified by the ants. Like in ACS [20], we allow only the best ant to deposit additional pheromones on the pairs $(s, l)$ belonging to its solution $x^{g b}$. The global update is done by applying the following global update rule:

$$
\tau(s, l)=(1-\alpha) . \tau(s, l)+\alpha . \Delta \tau(s, l)
$$

where

$$
\Delta \tau(s, l)= \begin{cases}\frac{1}{U\left(x^{g b}\right)} & \text { if }(s, l) \in x^{g b} \\ 0 & \text { otherwise }\end{cases}
$$

where $\alpha$, with $0<\alpha<1$, is the persistence of the trail so that (1- $\alpha)$ simulates the pheromone evaporation. Evaporation decreases the intensity as time goes and reduces the danger of premature convergence to poor local optima. $U\left(x^{g b}\right)$ is the energy function of the best solution found since the beginning of the algorithm (Eq.11). The best solution represent the optimal partition in which each pixel $s$ is mapped to its closest classes taking into account the class labels of it's immediate neighborhood. The pheromone values of the so-far best solution's pairs $(s, l)$ are increased to intensify the research of next solutions in a neighborhood of this solution during the next cycle by the future ants.

\subsection{Local improvement}

In order to improve the performance of ACS-MRF algorithm an additional local search is performed for each partition built by an ant. Adding a local search yields a faster convergence of the algorithm and an earlier detection of high quality solutions. Each created solution is locally improved before its evaluation. The local search algorithm start with a partition $x$ found by an ant and iteratively improves it using neighborhood search $N(x)$ and a search strategy [1]. The neighborhood $N(x)$ is a set of candidate partitions that can be reached from $x$ by making small modifications. The modifications should perform local finetuning towards a local optimum [1]. In ACS-MRF algorithm, we consider the first improving neighbor selection strategy. For each solution $x$, we evaluate the neighboring candidates solutions $x^{\prime} \in N(x)$ and the first $x$ ' for which $U\left(x^{\prime}\right)<U(x)$, ie. the first improving neighbor encountered, is selected. The neighborhood of a solution is defined by a set of solutions that can be obtained by exchanging the class labels of two pixels [38]. The sequential version of the local search algorithm is given in figure2. 
Algorithm 2 A local search algorithm

Let $x \in \mathrm{X}$ the segmentation to improve

Let $w=\phi$

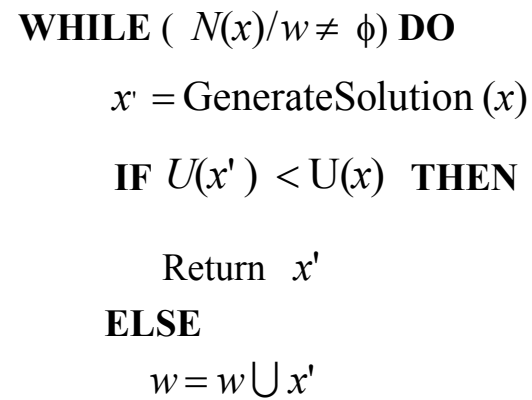

\section{END-WHILE}

Return $x$

Figure 2. The local search algorithm

\section{Experimental results}

To evaluate the performance of the proposed method other heuristics, we empirically compare the ACSMRF algorithm to the simulated annealing algorithm (SA) and the genetic algorithm (GA). All these algorithms have been implemented in Language C. Experiments were conducted by performing a variable number of runs for each algorithm with several parameters whose values are tabulated in Table 1.

\begin{tabular}{|ll|ll|ll|}
\hline \multicolumn{2}{|c|}{ Simulated annealing (SA) } & \multicolumn{2}{c|}{ Genetic algorithm (GA) } & \multicolumn{2}{c|}{ Ant colony system (ACS-MRF) } \\
\hline Parameters & Value & Parameters & Value & Parameters & Value \\
\hline$T_{0}$ & 2 & $\mathrm{~N}$ & 30 & $Q_{0}$ & 0.6 \\
$T_{m}$ & 0.9 & $P_{c}$ & 0.8 & $\tau_{0}$ & 0.001 \\
$N_{i}$ & 100 & $P_{m}$ & 0.01 & $\rho$ & 0.9 \\
$N_{\max }$ & 3000 & $N_{\max }$ & 1000 & $N_{\text {ants }}$ & 10 \\
& & & & $N_{\max }$ & 2500 \\
\hline
\end{tabular}

Table1. Parameters of the Simulated annealing, Genetic algorithm and Ant colony system algorithms. $T_{0}$ : initial temperature, $T_{m}$ : temperature multiplier, $N_{i}:$ number of iterations after which temperature is reduced, $N_{\max }$ : maximum number of iterations allowed, $N$ : the population size, $P_{c}$ : crossover probability,

$P_{m}$ : mutation probability, $q_{0}$ : parameter with determine the relative importance of exploitation versus exploration, $\tau_{0}$ : the initial pheromone trail, $\rho$ : the pheromone decay parameter and $N_{\text {ants }}$ : number of ants.

Experiments were conducted both on synthetic cerebral magnetic resonance (MR) images and on a real world mage represented by a house image.

\subsection{MR images}

Brain matter can generally be categorized as white matter (WM), gray matter (GM) and cerebrospinal fluid (CSF). The most problems with the segmentation of MR images are due to the presence of noise and to the intensity inhomogeneities. Assessing the performance of the image segmentation method is difficult when the ground truth is non-known. For this reason we used a computationally synthesized phantom available on the site Brainweb: http://www.bic.mni.mcgill.ca/brainweb/. The Brainweb site offers a large amount of different phantoms of MR brain images with different levels of noise and inhomogeneities. From 
these phantoms, the tissues classification in CSF, GM and WM are known a priori. Theses phantom images are labeled manually by a medical researcher and considered as the "ground truth" which is taken as a reference image and allows us to test the suggested algorithm. Tests have been done on the Brainweb phantoms (Figure 3 ) containing $0 \%, 3 \%$ or $5 \%$ of noise and have an inhomogeneity of $20 \%$ or $40 \%$. We tested each of SA, GA and ACS-MRF methods on each combination of these parameters for the Brainweb phantoms.

To validate the accuracy and reliability of the segmentation method, compared with the ground truth, we computed the Jaccard similarity, which measures the similarity of two sets as the ration of the size of their intersection divided by the size of their union. Let $V_{g}^{k}$ and $V_{s}^{k}$ denotes the total number of pixels labeled into a tissue class $k$ in the ground truth $(g)$ and the obtained segmentation $(s)$. For tissue class $k$ the Jaccard similarity $J^{k}(g, s)$ is defined by

$$
J^{k}(g, s)=\frac{\left|V_{g}^{k} \cap V_{s}^{k}\right|}{\left|V_{g}^{k} \cup V_{s}^{k}\right|}
$$

A good segmentation is obtained when $J^{k}(g, s)$ is near 1 which means that the tissue class $k$ of the brain is well detected. We computed the Jaccard similarity for each segmentation method. Table 2 shows obtained results. All values are average values over 10 test runs. Results show that ACS-MRF is completive with SA and GA and in most of the time, ACS found better solutions.

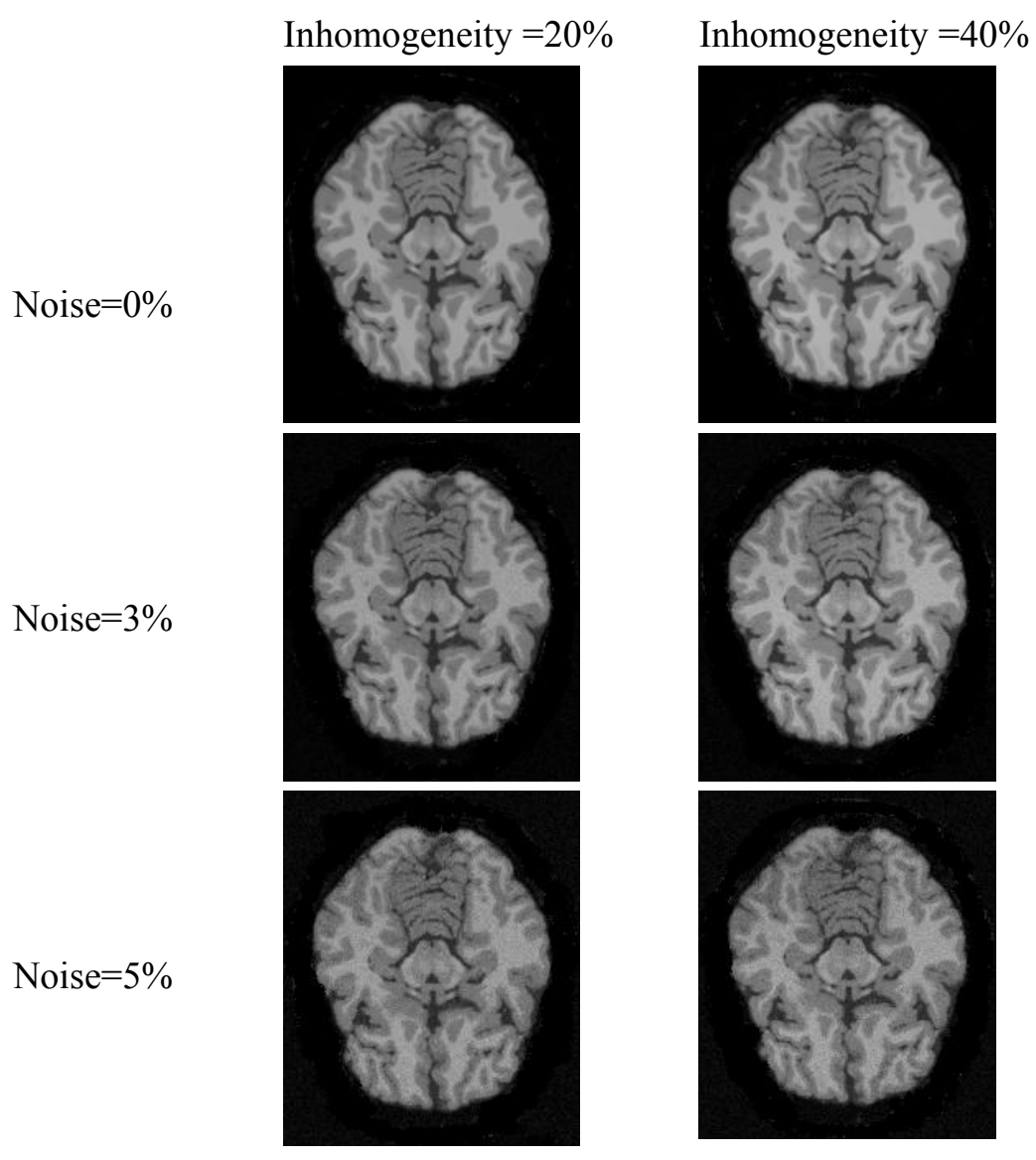

Figure 3. Brain phantoms with different values of noise and inhomogeneities 


\begin{tabular}{llllllll}
\hline \multirow{3}{*}{ SA } & & $\mathrm{N}=0 \%$ & & $\mathrm{~N}=3 \%$ & & $\mathrm{~N}=5 \%$ \\
\cline { 2 - 7 } & $20 \%$ & $40 \%$ & $20 \%$ & $40 \%$ & $20 \%$ & $40 \%$ \\
\hline \multirow{3}{*}{ GA } & LCR & 0.86 & 0.87 & 0.86 & 0.85 & 0.82 & 0.80 \\
& GM & 0.91 & 0.89 & 0.88 & 0.88 & 0.86 & 0.84 \\
& WM & 0.90 & 0.86 & 0.88 & 0.84 & 0.83 & 0.81 \\
\hline \multirow{3}{*}{ ACS-MRF } & LCR & 0.85 & 0.89 & 0.87 & 0.86 & 0.82 & 0.81 \\
& GM & 0.96 & 0.90 & 0.90 & 0.88 & 0.87 & 0.85 \\
& WM & 0.91 & 0.86 & 0.88 & 0.84 & 0.83 & 0.82 \\
\hline & GCR & 0.89 & 0.87 & 0.87 & 0.85 & 0.86 & 0.82 \\
& WM & 0.94 & 0.89 & 0.90 & 0.89 & 0.88 & 0.86 \\
& WM & 0.93 & 0.88 & 0.89 & 0.86 & 0.84 & 0.82
\end{tabular}

Table2. Comparison of Jaccard similarity measure of the SA, GA and ACS algorithms for segmentation of MR images.

\subsection{Real world image}

Our algorithm has been applied to a real world image represented by a house image. The experimental results are presented in Figure 4. It can be shown that the algorithm accurately segmented the regions of interest and produced more compact regions as a whole.
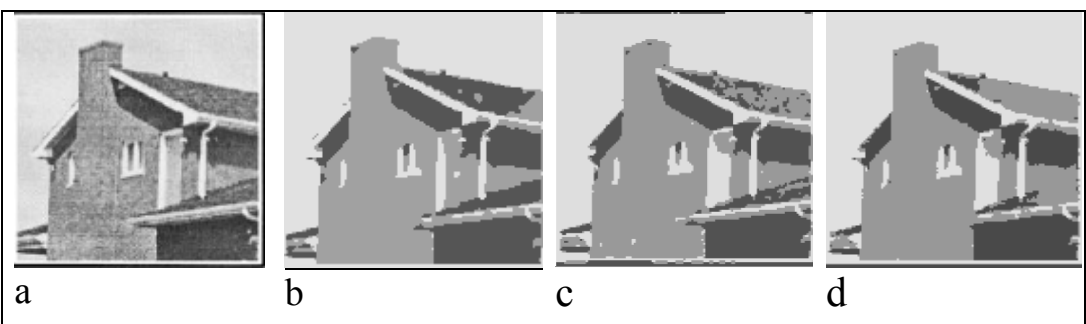

Figure 4. Results of segmentation of a house image. a) a house image. b) Result of SA algorithm, c) Result of the GA algorithm, d) Result of ACS-MRF algorithm.

\section{Conclusion}

In this paper, we have described a novel approach to image segmentation based on a hybrid Ant Colony System with a local search for the discrete optimization of the MRF-based image segmentation. MRF theory provides a convenient and consistent way to model contextual constraints among image pixels by conditional probability over small neighborhoods of pixels. The image is segmented by maximizing the a posteriori probability of the labeling space given the image pixels. The problem of finding the MAP estimate of the segmentation is expressed as the minimization of the posterior energy function. This minimization is combinatorial and so difficult to solve. ACS-MRF algorithm is a distributed algorithm based on a population of ants. Each ant constructs a candidate partition using the pheromone information accumulated by the others ants. After some iterations, the best partition representing the optimum value of the posterior energy function is found. A simple local search algorithm is used to improve the quality of the solution found by an ant and yielding a faster convergence of the algorithm. Experimental results show that the ACS-MRF based image segmentation is able to find high-quality solutions and is competitive with the other optimization methods like SA and GA. Two important directions for future research are as follows. First, it would be interesting to include a suitable heuristic function in conjunction with the pheromone trail and to investigate the performance of others kinds of pheromone updating strategy. Second, it would be interesting to study a parallel implementation of ACS-MRF algorithm to speed up the convergence process. 


\section{Acknowledgments}

We would like to thank Gianni Di Caro from IRIDIA, Université Libre de Bruxelles, for its valuable comments that improved the presentation of the paper.

\section{References}

[1] A. Franti, J. Kivijarvi, "Randomized Local Search Algorithm for the Clustering Problem", Pattern Analysis \& Applications (3): 358-369. 2000.

[2] A.H.S. Solberg, T. Taxt, A.K. Jain, "A Markov random field model for classification of multisource satellite imagery", IEEE Trans. Geoscience and Remote Sensing 4(32): 768-778, 1994.

[3] B. Bullnheimer, R. Hartl, C. Strauss, "Applying the Ant System to the Vehicle Routing Problems", 2nd Metaheuristic International Conference (MIC-97), Sophia-Antipolis, France, 1997.

[4] C. Kervrann, F. Heitz, "A Markov random field model based approach to unsupervised texture segmentation using local and global spatial statistics", IEEE trans. Image Process. (4), 1995.

[5] D. Costa., A. Hertz., "Ants Can Color Graphs", Journal of the Operational Research Society, (48): 295305, 1997.

[6] D.E. Goldberg, Genetic Algorithms in Search, Optimization, and Machine Learning Addision-Wesley, Reading, MA. 1989.

[7] D.K. Panjwani, G. Healey, "Markov random field models for unsupervised segmentation of textured color images", IEEE Trans. Pattern Anal. Machine Intell. 10(17): 939-954, 1995.

[8] E. Taillard, L. Gambardella, "An Ant Approach for Structured Quadratic Assignment Problems", $2^{\text {nd }}$ Metaheuristic International Conference (MIC-97), Sophia-Antipolis, France, 1997.

[9] E.Y. Kim, S.H. Park, H. J. Kim, „A Genetic algorithm-based segmentation of Markov Random Field modeled images", IEEE Signal processing letters 11(7): 301-303, 2000.

[10] F.T. Lin, C.Y. Kao, C.C. Hsu, "Applying the genetic approach to simulated annealing in solving some NP-hard problems", IEEE Trans. Syst. Man Cybernet. 6(23) : 1752-1767,1993.

[11] G. Di Caro, M. Dorigo, "AntNet: Distributed Stigmergetic Control for Communications Networks", Journal of Artificial Intelligence Research (JAIR), (9): 317-365, 1998.

[12] J. Besag, "On the statistical analysis of dirty pictures", J. Roy. Statist. Soc. B 3(48): 259-302, 1986.

[13] J. Gunnels, P. Cull, J.L. Holloway, "Genetic algorithms and simulated annealing for gene mapping”, In: Proc. 1st IEEE Conf. Evolutionary Computation, 385-390. 1994.

[14] J.H. Holland, Adaptation in Natural and Artificial Systems The University of Michigan Press, Ann Arbor, IL. 1975.

[15] J.L. Chen, A. Kundu, "Unsupervised texture segmentation using multichannel decomposition and hidden Markov-models", IEEE Trans. Image Process. 5(4) : 603-619, 1995.

[16] L. Gambardella, M. Dorigo, "HAS-SOP: An hybrid ant system for the sequential ordering problem", Technical Report (11), IDSIA, Lugano, CH. 1997.

[17] M. Dorigo, V. Maniezzo, A. Colorni, "Positive feed back as a search strategy", Technical Report (16) Politecnico di Milano, Italy. 1991.

[18] M. Dorigo, V. Maniezzo, A. Colorni, "The Ant System: Optimization by a Colony of Cooperating Agents", IEEE Transactions on Systems, Man and Cybernetics-Part B, 1(26): 29-41. 1996.

[19] M. Dorigo, M. Gambardella, "Ant Colony for the Traveling Salesman Problem", BioSystems, (43): 7381, 1997.

[20] M. Dorigo, L. Gambardella, "Ant Colony System: A Cooperative Learning Approach to the Traveling Salesman Problem”, IEEE Transactions on Evolutionary Computation (1): 53-66, 1997. 
[21] M. Dorigo, G. Di Caro, L. Gambardella,, “Ant Algorithms for Discrete Optimization”, Artificial Life. 2(5): 137-172, 1999.

[22] N.R. Pal, S.K. Pal, “A review on image segmentation techniques", Pattern Recognition 9(26): 12771294, 1993.

[23] P. Andrey, P. Tarroux, "Unsupervised segmentation of Markov random field modeled textured images using selectionist relaxation", IEEE Transactions on Pattern Analysis and Machine Intelligence, 3(20): 252-262, 1998.

[24] R. Beckers, J.L. Deneubourg, S.Goss, "trails and U-turns in the selection of the shortest path by the ant Lasius Niger", Journal of Theoretical Biology, (159):397-415, 1992.

[25] R. Chellappa, A.K. Jain, Editors, Markov Random Fields: Theory and Application Academic Press, San Diego, 1993.

[26] R. Dubes, S. Nadabar, C.C. Chen, A.K. Jain, "MRF Model-Based algorithm for Image Segmentation", Proc. 10th Int'l. Conference on Pattern Recognition, Atlantic City, 808-814, 1990.

[27] R.O. Duda. P.E. Hart, Pattern Classification and Scene Analysis. New Work: Wiley. 1973.

[28] S. Geman, D. Geman, "Stochastic relaxation, Gibbs distribution, and Bayesian restoration of images", IEEE Trans. Pattern Anal. Machine Intell. 6(6): 721-741, 1984.

[29] S. Krishnamachari, R. Chellappa, "Multiresolution Gauss-Markov random field models for texture segmentation". IEEE Trans. Image Process. 2(6): 251-267, 1997.

[30] S. Laskshmanan, H. Derin, "Simultaneous segmentation pg Gibbs Random field using Simulated Annealing”, IEEE Trans. On Pattern Analysis and Machine Intelligence. 8(11): 799-813. 1989.

[31] S.Z. Li, Markov Random Field Modeling in Computer Vision, Springer, Tokyo, 1995.

[32] T. Yamazaki, D. Gingras, "Image classification using spectral and spatial information based on MRF models", IEEE Trans. Image Processing 9(4): 1333-1338, 1995.

[33] U. Bhattacharya, B.B. Chaudhuri, S.K. Parui, "An MLP-based texture segmentation method without selecting a feature set", Image Vision Comput. 12(15): 937-948, 1997.

[34] V. Maniezzo, A. Colorni, M. Dorigo, "The Ant System Applied to the Quadratic Assignment Problem”, Technical Report. IRIDIA. (28), Université Libre de Bruxelles, Belgium, 1994.

[35] Y. Hu, T.J. Dennis, "Simulated annealing and iterated conditional modes with selective and confidence enhanced update schemes", In: Proc. 5th Annual IEEE Symp. Computer-based Medical Systems, $257-$ 264, 1992.

[36] Y. Huang, K. Palaniappan, X. Zhuang, J.E. Cavanaugh, "Optic flow field segmentation and motion estimation using a robust genetic partitioning algorithm", IEEE Tranactions and Pattern Analysis. Machine Intelligence. (17), pp. 1177-1190. 1995.

[37] Z. Kato, "Modélisation Markoviennes Multiresolutions en vision par ordinateur. Application à la segmentation d'images SPOT", Thèse de l'Université de Nice Sophia Antipolis, 1994.

[38] Z. Michalewicz, Genetic Algorithms +Data Structures =Evolution Programs, Springer-Verlag, Berlin, 1992. 